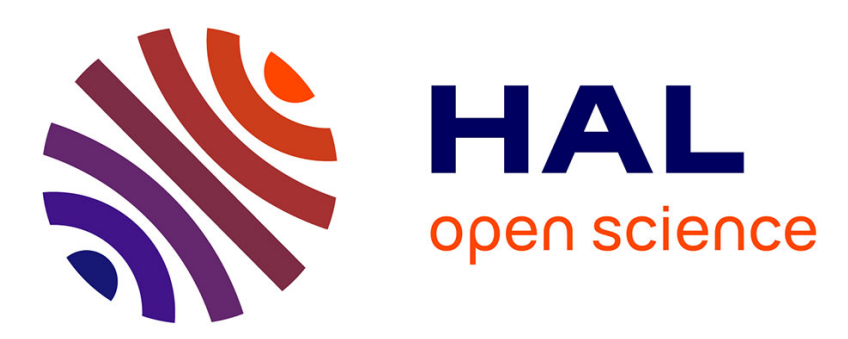

\title{
Modelization of electrical and optical characteristics of short-wave infrared type I InGaAsBi/InGaAs/InP quantum wells $p$-i-n detector
}

\author{
N Sfina, I Ammar, J.-L. Lazzari, M Said
}

\section{- To cite this version:}

N Sfina, I Ammar, J.-L. Lazzari, M Said. Modelization of electrical and optical characteristics of short-wave infrared type I InGaAsBi/InGaAs/InP quantum wells p-i-n detector. Physica Scripta, 2020, 96 (3), pp.035802. 10.1088/1402-4896/abd49a . hal-03144158

\section{HAL Id: hal-03144158 \\ https://hal.science/hal-03144158}

Submitted on 4 Mar 2021

HAL is a multi-disciplinary open access archive for the deposit and dissemination of scientific research documents, whether they are published or not. The documents may come from teaching and research institutions in France or abroad, or from public or private research centers.
L'archive ouverte pluridisciplinaire HAL, est destinée au dépôt et à la diffusion de documents scientifiques de niveau recherche, publiés ou non, émanant des établissements d'enseignement et de recherche français ou étrangers, des laboratoires publics ou privés. 


\title{
Modelization of electrical and optical characteristics of short-wave infrared type I InGaAsBi/InGaAs/InP quantum wells p-i-n detector
}

\author{
N. Sfina ${ }^{1,3, *}$, I. Ammar, J.-L. Lazzari ${ }^{2}$ and M. Said ${ }^{1}$ \\ ${ }^{1}$ Laboratoire de la Matière Condensée et des Nanosciences (LMCN), Département de Physique, Faculté des \\ Sciences de Monastir, Université de Monastir, Avenue de l'Environnement, 5019 Monastir, Tunisia. \\ ${ }^{2}$ Centre Interdisciplinaire de Nanoscience de Marseille (CINaM), UMR CNRS 7325 - Aix-Marseille Université, \\ Case 913, Campus de Luminy, 13288 Marseille cedex 9, France. \\ ${ }^{3}$ Faculty of science and art KKU Mahail Assir, king Kaled university Saoudi arabia
}

*Corresponding authors: Noureddine Sfina sfina fsm@yahoo.fr

\begin{abstract}
In the present work, we will exhibit a theoretical analysis and optimization of electrical and optical characteristics of a short-wave infrared p-i-n detector closely lattice matched to conventional (001) InP substrate by the use of quaternary dilute bismide alloy $\mathrm{In}_{\mathrm{x}} \mathrm{Ga}_{1-\mathrm{x}} \mathrm{As}_{1-\mathrm{y}} \mathrm{Bi}_{\mathrm{y}} / \mathrm{In}_{\mathrm{x}} \mathrm{Ga}_{1-\mathrm{x}} \mathrm{As}$ quantum wells as an active layer. The content of about $6 \%$ of Bismuth has been responsible of red-shift of the 50\% cut-off wavelength from 2.2 towards $2.8 \mu \mathrm{m}$ at room temperature, resulting in a band gap reduction of nearly $305 \mathrm{meV}$ caused by the bismuth incorporation. The temperature dependence of zero-bias resistance area product $\left(R_{0} A\right)$ and bias dependent dynamic resistance of the designed structure have been investigated thoroughly to analyses the dark current contributions mechanisms that might limit the electrical performance of the considered structure. It was revealed that the $R_{0} A$ product of the detector is limited by thermal diffusion currents when temperatures are elevated whereas the ohmic shunt resistance contribution limits it when temperatures are low. The modeled heterostructure, reveals a comforting dark current of $1.25 \times 10^{-8} \mathrm{~A}$ at bias voltage of $-10 \mathrm{mV}$ at $300 \mathrm{~K}$. The present work demonstrates that the p-i-n detector based on compressively strained $\operatorname{In}_{x} \mathrm{Ga}_{1-x} \mathrm{As}_{1-y} \mathrm{Bi}_{\mathrm{y}}$ quantum well is a potential candidate for achieving a short-wave infrared detection.
\end{abstract}

Keywords: Dilute-Bismide, Band Structure Engineering; Quantum Well p-i-n Heterostructure; Short-wave Infrared Detection; Dark Current; Dynamic resistance

\section{Introduction}


Bismide compounds have attracted a great attention throughout the world in the last few years and present nowadays a colossal challenge in semiconductor engineering devices. Indeed, thanks to their optical properties, they have the virtue to significantly reduce the band gap of containing semiconductor structures. This property makes the latter structures very promising in the purpose to realize infrared optoelectronic devices such as infrared emitters, infrared detectors, laser diodes, solar cells and spintronics [1-3]. Besides, on the energy plan, the incorporation of a minor quantity of Bi exalts the spin-orbit splitting energy which leads to a decay of the rate of Auger recombination and the inter-valence band absorption. These last properties are very advantageous for infrared optoelectronic devices [4]. The interest to InGaAsBi quaternary alloys has begun since several years with their growth by Feng et al $[5,6]$. They evidenced that the band gaps of such alloys are a temperature-insensitive. Petropoulos et al have shown later that the band gap energy of InGaAsBi is cut down by the incorporation of $\mathrm{Bi}$ with the rate of about $-50 \mathrm{meV} / \%$ $\mathrm{Bi}$ [7]. Also, beyond the gap reduction, the dramatic changes caused by the incorporation of a small fraction include modifications of responsivity and optical absorption coefficient [8]. Furthermore, two years ago, an infrared InGaAsBi detector grown on InP substrate was studied by $\mathrm{Gu}$ et al [9]. The former structure was provided with the quaternary InGaAsBi absorption layer leading to a $50 \%$ cut-off wavelength and to a bandgap $\backslash$ reduction of about $180 \mathrm{meV}$ at room temperature. In addition, $\mathrm{Gu}$ et al measured a dark current density of $2.4 \times 10^{-4} \mathrm{~A} / \mathrm{cm}^{2}$ at a bias voltage of $-10 \mathrm{mV}$ which is lower than what found in previous works giving perspectives for further better wavelength cut-off. Hence, it was demonstrated by Gu et al that the resistance area product $\mathrm{R}_{0} \mathrm{~A}$ of the detector increases as the temperature decreases. All these previous results make encouraging pushing forward research for better photodetector performance. These detectors have a large applications fields within remote sensing, environmental monitoring, and night vision $[10,11]$.

In this work, and in order to improve the performance of such detectors, we suggest enhancing optical excitonic absorption in the intrinsic zone by introducing a MQWs structure InGaAsBi/InGaAs/InP embedded between two strongly oppositely doped InP barrier layers. The whole structure is grown on InP substrate. We evoke here the good lattice match between InGaAsBi and InP that provides an advantage to such structures to be inserted in the active region of short-wave infrared detectors. This paper is ordered as follows: first, a quick introduction in Section 1 , in section 2 , we summarize the study of the quantum well structure and the 
optoelectronics properties. In Section 3, we introduce the suitable expressions in order to simulate the different mechanisms involved in the dark current, the associated resistance area product and the contribution of each one of these mechanisms. Then, in Section 4, we exhibit numerical calculations of the optical and electrical characteristics of the detector, results are performed and discussed. Ultimately, in Section 5, we provide conclusions about the entire work.

\section{Quantum Well design}

The modeled structure under investigation consists in two $\mathrm{In}_{0.53} \mathrm{Ga}_{0.47} \mathrm{As}$ layers separated by an $\mathrm{In}_{053} \mathrm{Ga}_{0.47} \mathrm{As}_{0.94} \mathrm{Bi}_{0.06}$ layer the three layers being embedded between relaxed InP. In order to determine quantum levels, the wave functions and associated parameters, we work in the frame of the effective mass approximation. We were forced to solve the one-dimensional of Schrödinger's wave equation according to the growth direction $\mathrm{z}$ assuming a steadily varying effective mass; a Ben-Daniel-Duke Hamiltonian is applied [12]. The modeling parameters such as the offsets in conduction and valence bands as well as effective masses used in the simulation are outlined in references $[13,14]$. In figure 1 we have illustrated the different subband edges energies in the conduction and valence bands $\mathrm{hh}_{1}$ and $\mathrm{e}_{1}$ as well as the position of the detection energies in the active layer $\mathrm{In}_{053} \mathrm{Ga}_{0.47} \mathrm{As}_{0.94} \mathrm{Bi}_{0.06} / \mathrm{In}_{0.53} \mathrm{Ga}_{0.47} \mathrm{As}$ for the $\mathrm{e}_{1}-\mathrm{hh}_{1}$ transitions, depending on quaternary $\mathrm{In}_{053} \mathrm{Ga}_{0.47} \mathrm{As}_{0.94} \mathrm{Bi}_{0.06}$ layer thickness. From figure 1a you can see that, the energy levels increase until $\operatorname{In}_{053} \mathrm{Ga}_{0.47} \mathrm{As}_{0.94} \mathrm{Bi}_{0.06}$ layer thickness $5.5 \mathrm{~nm}$ for electrons and heavy holes and then become stationary. It is also clear on figure $1 \mathrm{~b}$, that the detection energy decreases until $\mathrm{In}_{053} \mathrm{Ga}_{0.47} \mathrm{As}_{0.94} \mathrm{Bi}_{0.06}$ layer thickness $5.5 \mathrm{~nm}$ and then after this value become stationary. The InGaAsBi/InGaAs/InP quantum well detector was studied with a p-i-n structure, as shown in figure 2. The $\mathrm{p}-\mathrm{i}-\mathrm{n}$ device started with a $100 \mathrm{~nm}$-thick $\mathrm{n}^{+} \mathrm{InP}$ buffer layer strongly doped with Si to about $1 \times 10^{18} \mathrm{~cm}^{-3}$, which also represented as the etching top and bottom contact layer. This latter layer was grown at the usual InP growth temperature.

\section{Theoretical considerations}

The modeled heterostructure of $\operatorname{In}_{053} \mathrm{Ga}_{0.47} \mathrm{As}_{0.94} \mathrm{Bi}_{0.06} / \mathrm{In}_{0.53} \mathrm{Ga}_{0.47} \mathrm{As} / \mathrm{InP}$ has to be calculated selfconsistently. The Hamiltonian includes $V_{B}(z)$ represents the band discontinuities at the interfaces, $V_{X C}(z)$, the local exchange-correlation potential energy and Hartree potential obtained by solving Poisson's equation. The relative wave functions $\Psi_{i}$ and the eigenvalues $E_{i}$ are determined starting 
from the results of Schrodinger's equation solutions assuming the effective mass approximation [15].

$$
\begin{aligned}
& \left(-\frac{\hbar^{2}}{2 m_{0}} \frac{d}{d z}\left(\frac{1}{m(z)} \frac{d}{d z}\right)+V_{B}(z)+V_{H}(z)+V_{x c}(z)\right) \Psi_{i}(z)=E_{i} \Psi_{i}(z) \\
& \varepsilon_{0} \frac{d}{d z}\left[\varepsilon_{r}(z) \frac{d}{d z} V_{H}(z)\right]=e^{2}\left[N_{D}(z)-N_{A}(z)+p(z)-n(z)\right]
\end{aligned}
$$

The considered MQW-design heterostructure consists of five periods of intrinsic $\mathrm{In}_{053} \mathrm{Ga}_{0.47} \mathrm{As}_{0.94} \mathrm{Bi}_{0.06} / \mathrm{In}_{0.53} \mathrm{Ga}_{0.47} \mathrm{As}$ type I separated by a InP barrier. The thickness of the quaternary InGaAsBi layer is $5.5 \mathrm{~nm}$, and the thickness of InGaAs layer is $20 \mathrm{~nm}$. This active region is embedded between $100 \mathrm{~nm}$ InP relaxed layers. These latter InP layers are doped $\mathrm{n}$ and $\mathrm{p}$ $\left(\mathrm{N}_{\mathrm{A}}=\mathrm{N}_{\mathrm{D}}=1 \times 10^{18} \mathrm{~cm}^{-3}\right)$. Also, the investigated structure is optimally designed for $2.2 \mu \mathrm{m}$ detection. For simplicity, we overlook the effects of impurities or defects in the host lattice. The p-i-n device structure is presented in figure 3 that exhibits the calculated band diagrams corresponding to the valence and conduction bands with an absorption region consisting on 5 periods of $\operatorname{In}_{053} \mathrm{Ga}_{0.47} \mathrm{As}_{0.94} \mathrm{Bi}_{0.06} / \operatorname{In}_{0.53} \mathrm{Ga}_{0.47} \mathrm{As}$ MQW structure optimally designed for $2.2 \mu \mathrm{m}$ detection. Besides, the Fermi levels are plotted with their wave-functions at zero bias.

With the aim to simulate the dark current densities in the propounded hetrostrecture InGaAsBi/InGaAs/InP, five types of theoretical mechanisms models were highlighted respectively, generation-recombination $\left(\mathrm{J}_{\mathrm{GR}}\right)$ currents densities, diffusion currents densities $\left(\mathrm{J}_{\text {Diff }}\right)$, the trap assisted tunneling current, band-to-band tunneling currents densities $\left(\mathrm{J}_{\mathrm{BTB}}\right)$, currents densities $\left(\mathrm{J}_{\mathrm{TAT}}\right)$ and the tunneling currents densities through the barriers $\left(\mathrm{J}_{\mathrm{T}}\right)$, whereas the conduction currents densities were logically ignored for this MQWs InGaAsBi/InGaAs/InP heterostructure. These currents densities are calculated using equations, according to the procedure outlined in references [16-18].

$$
\begin{aligned}
& J_{\text {diff }}=n_{i}^{2} \sqrt{q k_{B} T}\left(\frac{1}{N_{A}} \sqrt{\frac{\mu_{e}}{\tau_{e}}}+\frac{1}{N_{D}} \sqrt{\frac{\mu_{h}}{\tau_{h}}}\right)\left(e^{\frac{q V}{k_{B} T}}-1\right) \\
& J_{G R}=\frac{q n_{i}}{2 \tau_{G R}} \sqrt{\frac{2 \varepsilon_{0} \varepsilon_{r}\left(N_{A}+N_{D}\right)}{q N_{A} N_{D}}} \sqrt{V_{t}}\left(e^{\frac{q V}{2 k_{B} T}}-1\right)
\end{aligned}
$$




$$
\begin{aligned}
& J_{T A T}=\frac{q^{2} m_{T} V M^{2} N_{t}}{8 \pi \hbar^{3}\left(E_{g}-E_{t}\right)} \exp \left(-\frac{4 \sqrt{2 m_{T}\left(E_{g}-E_{t}\right)^{3}}}{3 q \hbar F(V)}\right) \\
& J_{B T B}=\frac{q^{3} E V \sqrt{2 m_{e}}}{4 \pi^{2} \hbar \sqrt{E_{g}}} \exp \left(\frac{-4 \sqrt{2 m_{e}} E_{g}^{3 / 2}}{3 q E \hbar}\right) \\
& J_{T}=\frac{e m_{i}^{*} k_{B} T}{2 \pi^{2} \hbar^{3}} \int_{0}^{\infty} T(E, V) \operatorname{Ln}\left[\frac{1+\exp \left(E_{f}-E / k_{B} T\right)}{1+\exp \left(E_{f}-E-e V / k_{B} T\right)}\right] d E
\end{aligned}
$$

Furthermore, the dynamic resistance and area product $\left(\mathrm{R}_{\mathrm{d}} \mathrm{A}\right)$, where $\mathrm{A}$ is the area of the detector, is obtained by the reciprocal of the derivative of the current density with respect to each mechanism voltage according to Gopal et al. [19]:

$\frac{1}{\left(R_{d} A\right)}=\frac{d J}{d V}$

The diffusion, the generation recombination, the trap assisted tunneling and the band-to-band tunneling differential resistance of the dynamic resistance can be obtained as:

$$
\begin{aligned}
& R_{d i f}^{-1}=\left(\frac{q}{k_{B} T}\right)\left[\frac{q A n_{i}^{2}}{N_{D}}\left\{\frac{k_{B} T}{q} \frac{\mu_{h}}{\tau_{h}}\right\}^{1 / 2} \tanh \frac{d}{L_{h}}\right] \exp \left(\frac{q V}{k_{B} T}\right) \\
& {\left[R_{G-R}^{-1}\right]_{V \succ 0}=2 A_{G-R}\left[\frac{k_{B} T}{q V_{t}^{3 / 2}} \sinh \left(\frac{q V}{2 k_{B} T}\right)+\frac{1}{V_{t}^{1 / 2}} \cosh \left(\frac{q V}{2 k_{B} T}\right)\right]} \\
& {\left[R_{G-R}^{-1}\right]_{V \prec 0}=2 A_{G-R}\left[\frac{1}{V_{t}^{1 / 2}}+\frac{V}{2 V_{t}^{3 / 2}}\right]} \\
& \left(R_{T A T}\right)^{-1}=\frac{2 q^{3} A \pi^{2} m_{e} M^{2}}{h^{3}\left(E_{g}-E_{t}\right)} N_{T} \exp \left(-\frac{B}{V_{t}^{1 / 2}}\right)\left(1+\frac{B}{2 V_{t}^{1 / 2}}\right) \\
& \left(R_{B T B}\right)^{-1}=\frac{q^{3} A}{4 \pi^{2} \hbar^{2}} \sqrt{\frac{2 m_{e}^{*}}{E_{g}}} \exp \left(\frac{-4 \sqrt{2 m_{e}^{*}} E_{g}^{3 / 2}}{3 q \hbar}\right)\left[E+\frac{V}{2 V_{t}}\left(E+\frac{4 \sqrt{2 m_{e}^{*}} E_{g}^{3 / 2}}{3 q \hbar}\right)\right] \\
& A_{G-R}=\frac{q n_{t} A}{2 \tau_{g r}}\left[\frac{2 \varepsilon_{0} \varepsilon_{s}\left(N_{A}+N_{D}\right)}{q N_{A} N_{D}}\right]^{1 / 2}
\end{aligned}
$$




$$
B=\frac{8 \pi\left(2 m_{e}\right)^{1 / 2}\left(E_{g}-E_{t}\right)^{3 / 2}}{\left\{3 q h\left(\frac{2 q N_{D}}{\varepsilon_{0} \varepsilon_{s}}\right)^{1 / 2}\right\}}
$$

In the frame of the homojunction approximation, we can obtain the voltage dependent depletion region width $W_{\text {dep }}$ using the following expression [20-22].

$W_{\text {dep }}=\left[\frac{2 \varepsilon_{0} \varepsilon_{S}\left(N_{D}+N_{A}\right) V_{t}}{q N_{A} N_{D}}\right]$

$V_{d}=\frac{k_{B}}{q} \ln \left(\frac{N_{A} N_{D}}{n_{i}^{2}}\right)$

$n_{i}=2\left(\frac{2 \Pi}{h^{2}}\right)^{3 / 2}\left(m_{e} m_{h}\right)^{3 / 4} T^{3 / 2} \exp \left(\frac{-E_{g}}{2 k_{B} T}\right)$

Where $\mathrm{q}$ is the electron charge, $\mathrm{N}_{\mathrm{A}}$ and $\mathrm{N}_{\mathrm{D}}$ are, respectively, the $\mathrm{p}$ and $\mathrm{n}$-type doping concentrations, $\mathrm{T}$ is the temperature, $\mu_{\mathrm{e}}, \tau_{\mathrm{e}}$ and $\mu_{\mathrm{h}}, \tau_{\mathrm{h}}$ are the mobilities and lifetimes of electrons and holes, respectively, $\tau_{\mathrm{GR}}$ is the generation-combination lifetime, $\varepsilon_{s}$ is the static dielectric constant, $\varepsilon_{\mathrm{r}}$ is the relative effective dielectric constant value of the multilayer $\mathrm{InGaAsBi} / \mathrm{InGaAs} / \mathrm{InP}, \varepsilon_{0}$ is the permittivity of free space, $V_{t}=V_{d}-V$ is the total junction potential, $\mathrm{V}$ is the bias voltage, $\mathrm{V}_{\mathrm{d}}$ is the built-in potential of the heterostructure, $\mathrm{k}_{\mathrm{B}}$ is the Boltzmann constant, $\mathrm{F}$ is the applied electric field, $\hbar$ is the Planck constant, $\mathrm{m}_{\mathrm{T}}$ is the reduced tunneling effective mass, $\mathrm{E}_{\mathrm{g}}$ is the band gap, $\mathrm{d}$ is the thickness of the $\mathrm{n}$ region, $L_{h}$ is the hole diffusion length and $\mathrm{N}_{\mathrm{T}}$ is the activated trap density which is given below :

$$
N_{T}=\frac{\left\{\left(\frac{\partial^{2} I_{d i f}}{\partial V^{2}}\right)+\left(\frac{\partial^{2} I_{g r}}{\partial V^{2}}\right)\right\}_{V=V_{m}}}{\frac{2 q^{3} A \pi^{2} m_{e} M^{2}}{h^{3}\left(E_{g}-E_{t}\right)} \exp \left(-\frac{B}{V_{t}^{1 / 2}}\right)_{V=V_{m}}\left(\frac{B}{4 V_{t}^{1 / 2}}+\frac{B^{2}}{4 V_{t}^{2}}\right)_{V=V_{m}}}
$$

$E_{t}$ is the trap energy location, $n_{i}$ represents the intrinsic carrier concentration inside the quantum well $\mathrm{InGaAsBi} / \mathrm{InGaAs} / \mathrm{InP}, \mathrm{M}^{2}$ is a matrix element associated with the trap potential and $\mathrm{V}_{\mathrm{m}}$ corresponds to the value of applied voltage $\mathrm{V}$ at the peak position. In table 1, we summarize the essential parameters adopted in this simulation work.

The whole dynamic resistance $R d$ of the considered structure is compounded by all the contributing impedances and is given by the expression [23]. 
$\frac{1}{R_{d}}=\frac{1}{R_{\text {Diff }}}+\frac{1}{R_{G-R}}+\frac{1}{R_{T A T}}+\frac{1}{R_{B T B}}$

The condition

$\frac{\partial R_{d}}{\partial V}=0$

corresponds to the peak position in $R_{d^{-}} V$ characteristics

\section{Results and discussion}

In this part, we display the results of our theoretical exploration of the structural, electrical, and optical characterizations of $\mathrm{InGaAsBi} / \mathrm{InGaAs} / \mathrm{InP}$ p-i-n heterostructure. To complete electrical characterizations, we studied the variation of the capacitance versus the reverse bias voltage $\mathrm{C}-\mathrm{V}$. Figure 4 illustrations the capacitance of the detector versus the reverse bias voltage, calculated using the following formula at a frequency of $1 \mathrm{MHz}$ :

$$
\left(\frac{A}{C(V)}\right)^{2}=\frac{2 V_{d}}{q \varepsilon_{r} \varepsilon_{0} N_{r e d}}-\frac{2}{q \varepsilon_{r} \varepsilon_{0} N_{r e d}} V
$$

where $A$ is the area of the structure, $V$ is the reverse bias voltage, the reduced carrier concentration $\mathbf{N}_{\text {red }}$ and the other parameters have already been defined. The electron concentration is around $0.6-1 \times 10^{17} \mathrm{~cm}^{-3}$ along the InGaAsBi absorption layer due to the impurities engendered by the growth temperature fluctuation. furthermore, the slightly elevated electron concentration in the InGaAsBi layer may be due to Bi-related defects [24]. By comparison with the results of the literature described in reference [25], we note that the capacitance is higher in the MQW active region then the active region formed by the $\mathrm{InGaAsBi}$ absorption layer. Moreover, the capacitance increases as the number of quantum wells increases.

In Figure 5, we show the calculated I-V characteristics at temperatures starting from 160 $\mathrm{K}$ till $300 \mathrm{~K}$ on $100 \mu \mathrm{m}$ diameter of detector are represented. At Room Temperature, notable electrical properties were found. Among them, a usual dark-current of $1.1410^{-7} \mathrm{~A}$ at $-1 \mathrm{~V}$ and a good differential resistance area product $\left(R_{0} A\right)$ reaching the value of $3.0710^{5} \Omega . \mathrm{cm}^{2}$. when temperature decreases, the dark current drops to $2.0710^{-9} \mathrm{~A}\left(R_{0} A=3.5210^{7} \Omega . \mathrm{cm}^{2}\right)$ at $200 \mathrm{~K}$. we note that he dark currents are inferior than those obtained earlier on wavelength-extended InGaAs detectors without $\mathrm{Bi}$ incorporation [26]. At reverse voltage the adoption of MQWs as the active region decreases the dark current while it increases the serial resistance by comparison with other devices used bulk InGaAsBi as active region [9, 27]. 
In order to comprehend the evolution of the dark current with the bias voltage, it is required to be aware of the eventual impact of each component issued from the different considered mechanisms that including the diffusion, generation-recombination, band-to-band tunneling, the trap assisted tunneling current, the tunneling currents densities through the barriers and thermally activated tunnel currents. The determination of every current component has been performed out at room temperature using the approach evoked in the preceding section. In figure 6, results are showed. According to our calculated results, it is noted that the characteristic of direct polarization is described by the thermal diffusion and the well while the behavior of the total dark current under inverse polarization is quite in agreement with the sum of the tunnel current and recombination contributions.

It is known that the dark current indicates the optical receiver sensitivity, it also indicates about the material quality. Recent studies have suggested a fruitful comparison with the experimental results [28], it was proved that our proposed structure is suitable to improve the performance of the detector by the incorporation of the Bi content in the absorption region.

To highlight the effect of temperature variation on the dark current and make it clear, we studied the Arrhenius plots in Figure 7 for reverse bias voltages varying between 0.01 until 1.5V. The elevated temperature dependence of dark current due to the effective thermal activation energy is given by the Arrhenius relation:

$$
I_{d} \propto \exp \left(-E_{a} / k_{B} T\right)
$$

Where $E_{a}, T$, and $k_{B}$ designate, respectively, the activation energy, the temperature, and Boltzmann's constant. The evolution of the curve of the profile of the product $\mathrm{R}_{0} \mathrm{~A}$ function of $(1000 / \mathrm{T})$ presented on Figure 7, indicates that the dark current is ruled by four different mechanisms according to the variation of the temperature regimes. Considering the temperature range between 160 and $180 \mathrm{~K}$, the p-i-n structure efficiency is limited by the temperatureinsensitive barriers tunneling effect [29]. For higher temperatures than $180 \mathrm{~K}$, generationrecombination (G-R) current is created by thermally generated carriers who starts to be diffusion stream limit at a temperature of $220 \mathrm{k}$. From 180 to $220 \mathrm{~K}$, the structure performance is controlled by the G-R process through an interesting recombination lifetime in the depleted region for temperatures above $210 \mathrm{~K}$, the diffusion current becomes the leading component at high temperature $220 \mathrm{~K}$. In this temperature range, the device performance is approaching to 
conventional III-V semiconductors devices without bismuth [30]. In the temperature range higher than $300 \mathrm{~K}$, the thermal recombination current seems to be the main dark current mechanism. At $0.01 \mathrm{~V}$ reversed bias, the activation energy is around $0.39 \mathrm{eV}$ at higher temperatures than $210 \mathrm{k}$. This shows that the dark current mechanism is limited by both G-R and diffusion process. For temperatures range below $200 \mathrm{~K}$, the activation energy is about $0.22 \mathrm{eV}$, which is nearly the half of the absorption layer strained band gap. The resistance area product $\mathrm{R}_{0} \mathrm{~A}$ function of reciprocal temperature is presented in Figure 7. At $300 \mathrm{~K}$, the value of the product $\mathrm{R}_{0} \mathrm{~A}$ of the detector is $39.12 \times 10^{3} \Omega . \mathrm{cm}^{2}$, the $\mathrm{R}_{0} \mathrm{~A}$ increases as the temperature decreases.

In Figure 8, we exhibit the results of the calculations of the associated dynamic resistance function of the bias voltage $\left(\mathrm{R}_{\mathrm{d}}-\mathrm{V}\right)$. Also, on the same figure we isolate the relative contribution of each mechanism. Furthermore, we note that the TAT constituent is a suitable approximation at reverse bias while $R_{d}-V$ whereas the characteristics $R_{d}-V$ are agreeably described by diffusion and G-R components at direct bias voltage at Room Temperature. The trap-assisted component is naturally increasing while the diffusion component is naturally decreasing, these properties enhance the peak of $R_{d}$. Indeed, as it is known, the $R_{d}-V$ characteristics of the diode exhibits a peak when the reverse bias voltage is low or medium in the case of contribution of TAT in the the dark current Gopal et al. [16]. We have estimated the trap density $\mathrm{N}_{t}$ which contributes in TAT using the position of this peak. As illustrated in Figure 8, we note that the differential resistance displays a peak which depends on temperature and spreads at elevated reverse bias voltage when temperature rises. The $\mathrm{R}_{\mathrm{d}}-\mathrm{V}$ peak at $300 \mathrm{~K}$ is superior than $10^{4} \Omega$ at $0 \mathrm{~V}$ and nearly $2.15 .104 \Omega$ at a reverse bias voltage of $150 \mathrm{mV}$. Discrepancies of the $\mathrm{R}_{d}-\mathrm{V}$ with temperature variations and applied bias show that at Room Temperature, the peak $R_{d}$ is limited by G-R and diffusion processes. The great value of dynamic resistance would enhance the detectivity in the considered wavelength range, which is advantageous in our work. Nevertheless, a large value of TAT current results in breakdown of the resistance area product $\mathrm{R}_{0} \mathrm{~A}$ of the detector even at restrained values of applied reverse voltage. This could be responsible of a collapse, at room-temperature, of detectivity of the considered device. Moreover, we can conclude about the material quality using the dark current which also is relevant to terminate about the optical receiver sensitivity. If we compare the experimental results pointed out to recent investigations [31] we could note that our considered structure allows improving the performance of the photodiode. 
In order to investigate the potential application of InGaAsBi/InGaAs/InP p-i-n heterostrcture. We have calculated the optical responsivity achieved with the use the following expression:

$$
R(\lambda)=\frac{\eta_{i} q \lambda \Omega}{h c} \frac{\Gamma \alpha(\lambda, V)}{\Gamma \alpha(\lambda, V)+\alpha_{i}}\left(1-\exp \left(-\left(\alpha_{i}+\Gamma \alpha(\lambda, V)\right) L\right)\right.
$$

where $\eta_{\mathrm{i}}$ represents the internal quantum efficiency, $\Gamma$ is the confinement factor of the optical mode within the multiple quantum wells structure, $\alpha(\lambda, V)$ refers to the absorption coefficient at wavelength $\lambda$ and bias $\mathrm{V}, \mathrm{L}$ is the waveguide length, and $\Omega$ is the coupling efficiency, including mode size mismatch and reflection [30] $\alpha_{i}\left(\alpha_{i}=120 \mathrm{~cm}^{-1}\right)$ represents all propagation losses that do not generate photocurrent. The quantum efficiency $\eta_{i}$ is correlated to absorption coefficient $\alpha$ and thickness $d$ of the absorption layer $\left(\eta_{i}=1-\exp (-\alpha d)\right)$. We have theoretically calculated the coefficient $\alpha(\lambda, V)$ in accordance with the formula outlined in our antecedent work [31]. At room temperature RT, the responsivity has been calculated for different reverse bias voltages $(0 \mathrm{~V},-1 \mathrm{~V}$ and $-2 \mathrm{~V})$ whose the results are exhibited in figure 9. This responsivity versus wavelength $(1.55 \mu \mathrm{m}-3 \mu \mathrm{m})$ spreads a peak. This peak corresponds to the $\mathrm{e}_{1}$ $\mathrm{hh}_{1}$ transition energy and the strained direct band gap of $\operatorname{In}_{053} \mathrm{Ga}_{0.47} \mathrm{As}_{0.94} \mathrm{Bi}_{0.06} / \mathrm{In}_{0.53} \mathrm{Ga}_{0.47} \mathrm{As} \mathrm{QWs}$; are observed around $2200 \mathrm{~nm}$. By the same token, we can easily note that the responsivity reduces when the reverse bias voltage is elevated. In the case of a reverse bias voltage of $-2 \mathrm{~V}$, we obtain the responsivities of $0.833 \mathrm{~A} / \mathrm{W}$ at $2200 \mathrm{~nm}$ at room temperature. Consequently, InGaAsBi/InGaAs heterostructures are a relevant competitor material either for all telecommunication bands in optoelectronics or shortwave infrared applications.

In addition to the dark current, we could examine the performance of MQW detector using its detectivity $\mathrm{D}^{*}$ given by the B.L Sharma basic formula, which varies by the incident light wavelength $\lambda$ :

$$
D^{*}(\lambda)=\frac{1}{2} R(\lambda)\left(q J_{0}\right)^{-\frac{1}{2}}
$$

where $\lambda$ is the considered wavelength and $\mathrm{J}_{0}$ is the dark current density. By using this simple formula in the area of zero bias voltage of p-n junction, the specific detectivity at a $2200 \mathrm{~nm}$ wavelength peak of responsivity has been calculated at $300 \mathrm{~K}$. The detectivity $\mathrm{D}^{*} \sim 1.9210^{8} \mathrm{~cm}$ 
$\mathrm{Hz}^{1 / 2} / \mathrm{W}$ and $1.6210^{8} \mathrm{~cm} \mathrm{~Hz}^{1 / 2} / \mathrm{W}$ have been attained under reverse bias voltage of $-0.5 \mathrm{~V}$ and $-1 \mathrm{~V}$, respectively, at Room Temperature RT.

In addition to optical communication, this type of devices would also be suitable for detection applications because of their higher electro-optical characteristics, specifically low dark current, greater sensitivity, high quantum efficiency and swift response.

\section{Conclusion}

In this paper, we have introduced a novel alternative active region based on the type-I transition of InGaAsBi/InGaAs/InP quantum wells detector, whose the structure is nearly latticematched to InP substrate was proposed and studied. In the considered structure, the Bi content is nearly $6 \%$ in the absorption layer and the cut-off wavelength has been extended to $2.8 \mu \mathrm{m}$ at Room Temperature RT, corresponding to a Bi-induced band gap reduction. The dark currents are lower than the other detectors taken InGaAsBi as absorption layer, which is very promising to improve the performance of $\mathrm{InGaAsBi} / \mathrm{InGaAs}$ quantum wells system. We have computed the electrical characteristics (resistance area product and dark current) and optical characteristics (responsivity) of the considered structure at RT and bias voltages. It is capital to point out the investigations of carrier transport mechanisms across the modeled junction. consequently, it is prominent to achieve a low dark current with a arguable photocurrent at elevated temperature. Hence, we have carried out an investigation about the initial dark current. The modelization takes into account five mechanisms processes. These results give to our considered and studied structure an auspicious potential of InP-based InGaAsBi/InGaAs detectors for shortwave infrared applications. 


\section{References}

[1] T. Takamoto, M. Kaneiwa, M. Imaizumi, and M. Yamaguchi, Prog. Photovoltaics,13, 495, (2005).

[2]: K. Bertulis, A. Krotkus, G. Aleksejenko, V. Pacebutas, and R. Adomavicius, Appl. Phys. Lett , 88, 201112, (2006).

[3] S. Francoeur, M.-J. Seong, and A. Mascarenhas, Appl. Phys. Lett., 82, 3874, (2003)

[4] B. Fluegel, S. Francoeur, A. Mascarenhas, S. Tixier, E. C. Young, and T. Tiedje, Phys. Rev. Lett., 97, 067205, (2006).

[5] G. Feng, M. Yoshimoto, K. Oe, A. Chayahara, and Y. Horino, Jpn. J. Appl. Phys., 44, 1161, (2005).

[6] G. Feng, K. Oe, and M. Yoshimoto, J. Cryst. Growth, 2007, 302, 121.

[7] J. P. Petropoulos, Y. Zhong, and J. M. O. Zide, Appl. Phys. Lett. , 99, 031110, (2011).

[8] Zhize Zhou, Danuta F Mendes, Robert D Richard, Faebian Bastiman and John PR David, Semicond. Sci. Technol 30; 94004, (2015)

[9] Y. Gu, Y. G. Zhang, X. Y. Chen, Y. J. Ma, S. P. Xi, B. Du and Hsby. Li, Appl. Phys. Lett., 108, 032102, (2016).

[10] R. W. M. Hoogeveen, R. J. Van Der A, and A. P. H. Goede, Infrared Phys. Technol, 42, 1, (2001).

[11] J. Davenson, V. Pacebutas, R. Butkute, A. Baranov, and A. Krotkus, Appl. Phys. Express, 5, 15503, (2012).

[12] N. Sfina, J.L. Lazzari, J. Derrien, F.A. d'avitaya, M. Said, Eur. Phys. J. B, 48, 151, (2005).

[13] X. Y. Chen, Y. Gu, Y. G. Zhang,a S. P. Xi, B. Du, Y. J. Ma, W. Y. Ji, and Y. H. Shi Aip Advances, 6, 075215, (2016).

[14] S. Jin and S. J. Sweeney, J. Appl. Phys, ,114, 213103, (2013).

[15] N. Yahyaoui, N. Sfina, J.-L. Lazzari, A. Bournel and M. Said. , Semicond. Sci.

Technol. 30, 085016, (2015).

[16] Q. K. Yang, F. Fuchs, J. Schmitz, and W. Pletschen, Appl. Phys. Lett. 81, 4757 (2002).

[17] J. Y. Wong, IEEE Trans. Electron Devices ED-27, 48 (1980).

[18] M. A. Kinch, J. Vac. Sci. Technol. 21, 215 (1982).

[19] V. Gopal, E. Plis, J. B. Rodriguez, C. E. Jones, L. Faraone, and S. Krishna, J. Appl. Phys. 104, 124506 (2008).

[20] Q. K. Yang, F. Fuchs, J. Schmitz, and W. Pletschen, Appl. Phys. Lett. 81, 4757 (2002).

[21] H. Mohseni, A. Tahraoui, J. S. Wojkowski, and M. Razeghi, Proc. SPIE 3948, 145 (2000).

[22] H. Mohseni, J. S. Wojkowski, A. Tahraoui, and M. Razeghi, Proc. SPIE 3948, 153 (2000).

[23] V. Gopal, S. Gupta, R. K. Bhan, R. Pal, P. K. Chaudhary, and V. Kumar, 
Infrared Phys. Technol. 44, 143 (2003).

[24] J. P. Petropoulos, Y. Zhong, and J. M. O. Zide, Appl. Phys. Lett. 99, 031110, (2011).

[25] Y. Gu, Y. G. Zhang, X. Y. Chen, Y. J. Ma, S. P. Xi, B. Du, and Hsby. Li, Appl Phys Letters 108, 032102, (2016)

[26] Y. G. Zhang, Y. Gu, C. Zhu, G. Q. Zhu, A. Z. Li, and T. D. Liu, Infrared Phys. Technol. 47, 257 (2006).

[27] Tonkikh A A, Eisenschmidt C, Talalaev V G, Zakharov N D, Schilling J, Schmidt G and Werner. PAppl. Phys. Lett. 103, 032106, (2013)

[28] Zhang D, Xue C, Cheng B, Su S, Liu Z, Zhang X, Zhang G, Li C and Wang Q Appl. Phys. Lett 102, 141111, (2013)

[29] D. Ahn, S.L. Chuang, IEEE J. Quantum Electron. 23 (1987) 2196

[30] X. Ji, B. Liu, Y. Xu, H. Tang, X. Li, H. Gong, B. Shen, X. Yang, P. Han, and F. Yan J App Physics 114, 224502, (2013).

[31] Masahiro Yoshimoto. Applications of Bismuth-Containing III-VSemiconductors in Devices. Molecular Beam Epitaxy, , 381, 394, (2019)

[32] Yahyaoui N, Sfina N, Abdi-Ben Nasrallah S, Lazzari J-L and Said M Comput. Phys. Commun. 185 3119, (2014)

[33] Ando Y and Itoh T J. Appl. Phys. 61 1497, (1987) 


\section{Figure captions}

Figure 1: (a) Electron and Heavy hole confinement-energies for the quaternary $\mathrm{InGaAsBi} / \mathrm{InGaAs} / \mathrm{InP}$ quantum wells heterostructure as a function of InGaAsBi layer thickness (b) Room temperature evolution of the detection energies and the wavelength of radiation corresponding to the edge of direct-band transitions of $\mathrm{e}_{1}-\mathrm{hh}_{1}$ versus the InGaAsBi layer thickness in $\mathrm{InGaAsBi} / \mathrm{InGaAs} / \mathrm{InP}$ heterostructure.

Figure 2: (a) Schematic structure of the InGaAsBi/InGaAs/InP p-i-n detector (b). Schematic diagram of a $\mathrm{p}-\mathrm{i}-\mathrm{n}$ detector device. The cross-sectional view shows the structure of strained InGaAsBi/InGaAs/InP multiple quantum wells grown on InP relaxed buffers.

Figure 3: The calculated band diagrams of the conduction and valence bands with an absorption region consisting on five periods of $\operatorname{In}_{053} \mathrm{Ga}_{0.47} \mathrm{As}_{0.94} \mathrm{Bi}_{0.06} / \mathrm{In}_{0.53} \mathrm{Ga}_{0.47} \mathrm{As} / \mathrm{InP} \mathrm{MQW}$ p-i-n structure optimally designed for $2.2 \mu \mathrm{m}$ detection. The Fermi levels are drawn with their wave functions at zero bias.

Figure 4: Capacitance as a function of reverse bias voltage of the detector calculated versus the number of quantum well in the absorption layer.

Figure 5: Dark current density of the p-i-n heterojunction based on InGaAsBi/InGaAs/InP MQW structure of $100 \mu \mathrm{m}$ in diameter versus bias voltage for temperatures from 160 to $300 \mathrm{~K}$. The current is nearly symmetric to zero voltage.

Figure 6: Total dark current density at $300 \mathrm{~K}$ as a function of bias voltage from $-2 \mathrm{~V}$ to $0.5 \mathrm{~V}$ is shown with three distinct regimes with different dominant mechanisms, the diffusion current, Generation-recombination current, Trapp assisted tunneling current.

Figure 7: Arrhenius plots of the dark current at $-0.01 \mathrm{~V}$ to $-1.5 \mathrm{~V}$ and zero bias resistance area products $\mathrm{R}_{0} \mathrm{~A}$ versus reciprocal temperatures.

Figure 8: Different mechanisms of dynamic resistance versus applied bias voltage at $300 \mathrm{~K}$.

Figure 9: Optical responsivity from $\lambda=1555 \mathrm{~nm}$ up to $\lambda=3000 \mathrm{~nm}$ for the $\mathrm{InGaAsBi} / \mathrm{InP}$ p-i-n detectors at $0,-1$ and $-2 \mathrm{~V}$, respectively. 

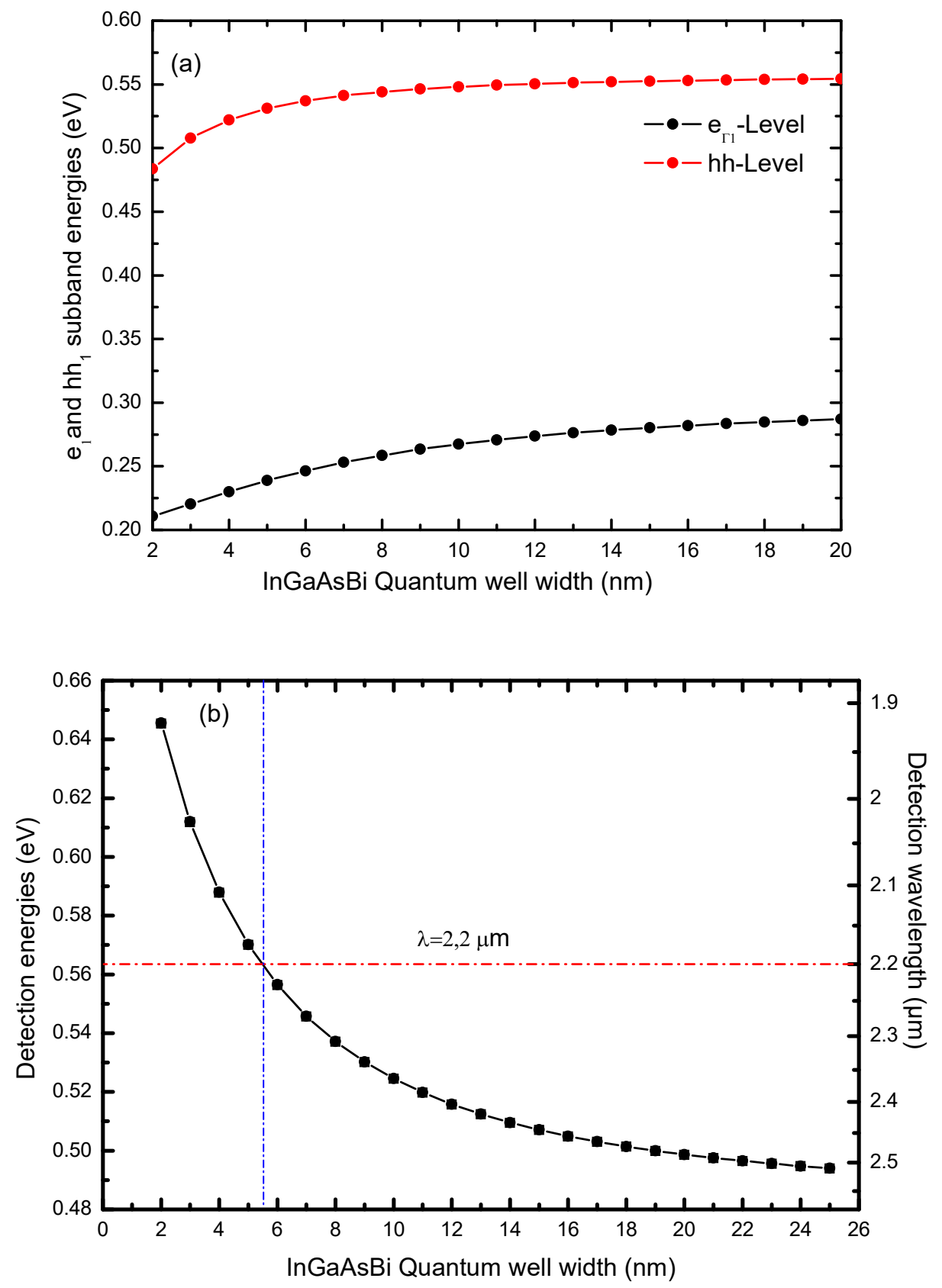

Figure 1 
(a)

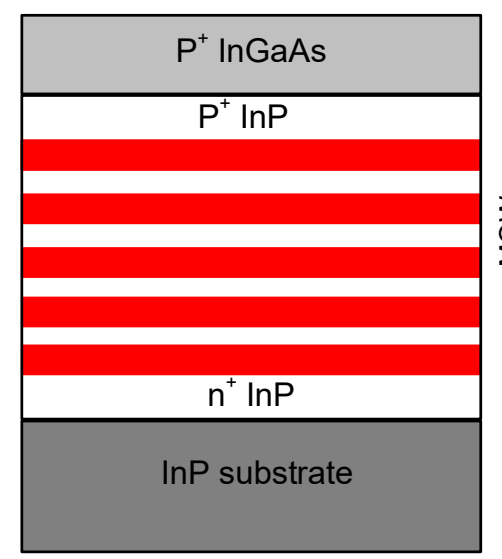

(b)

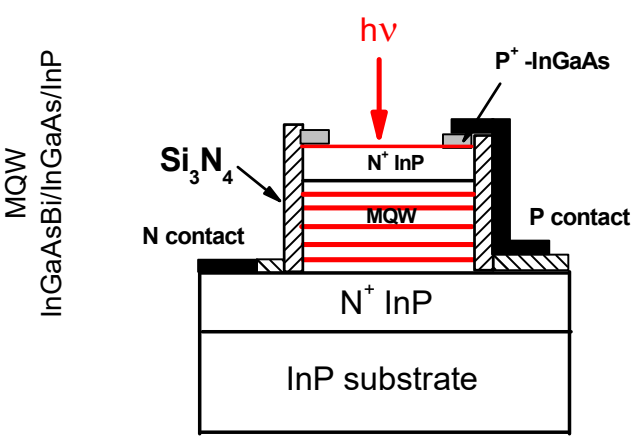

Figure 2

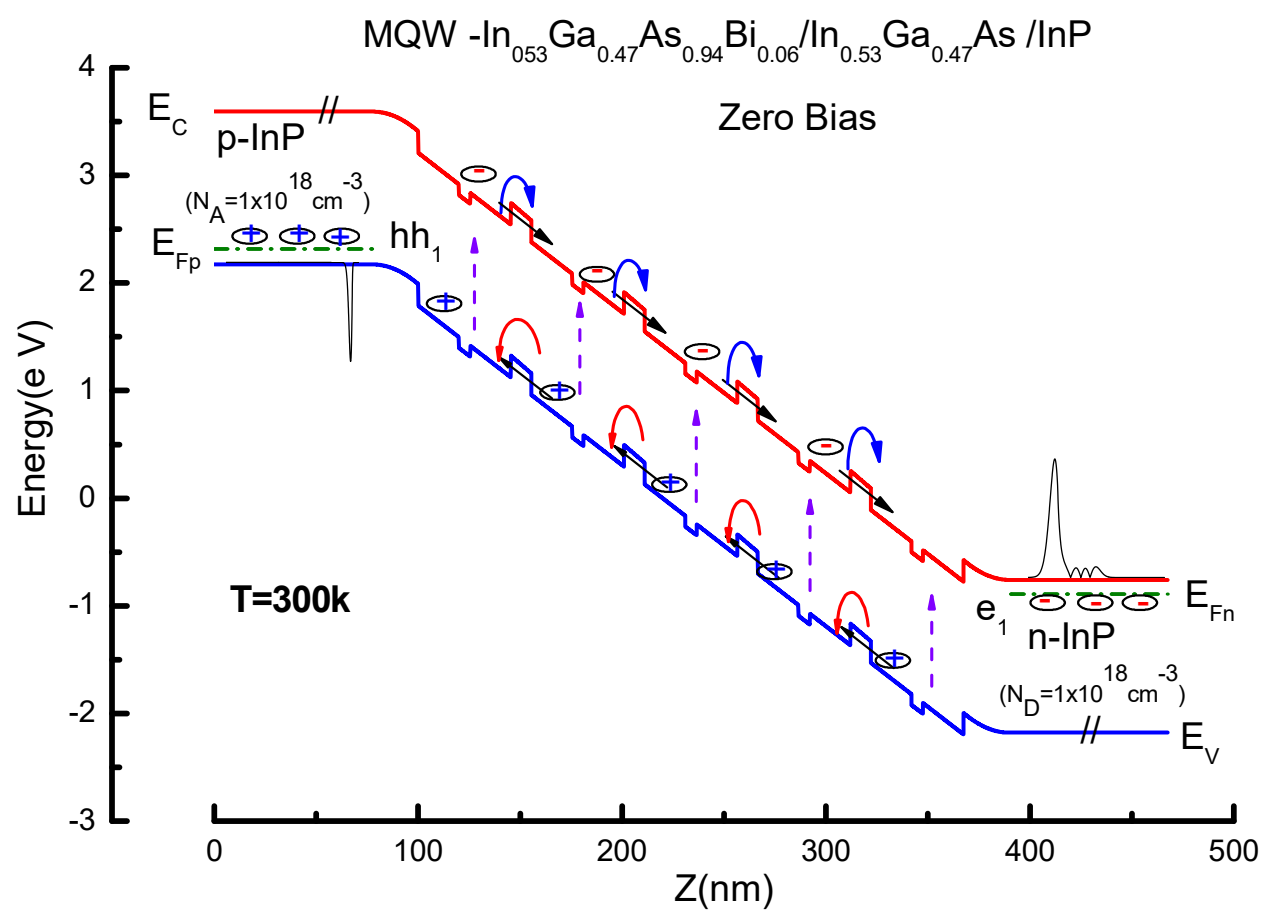

Figure 3 


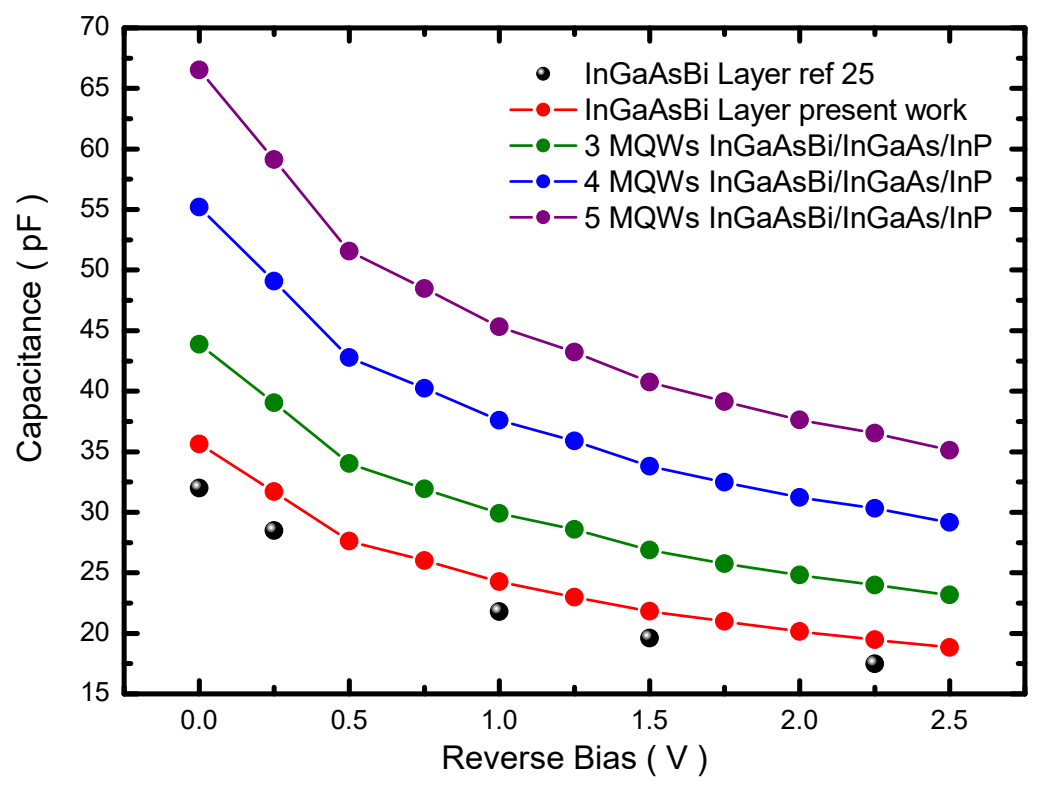

Figure 4

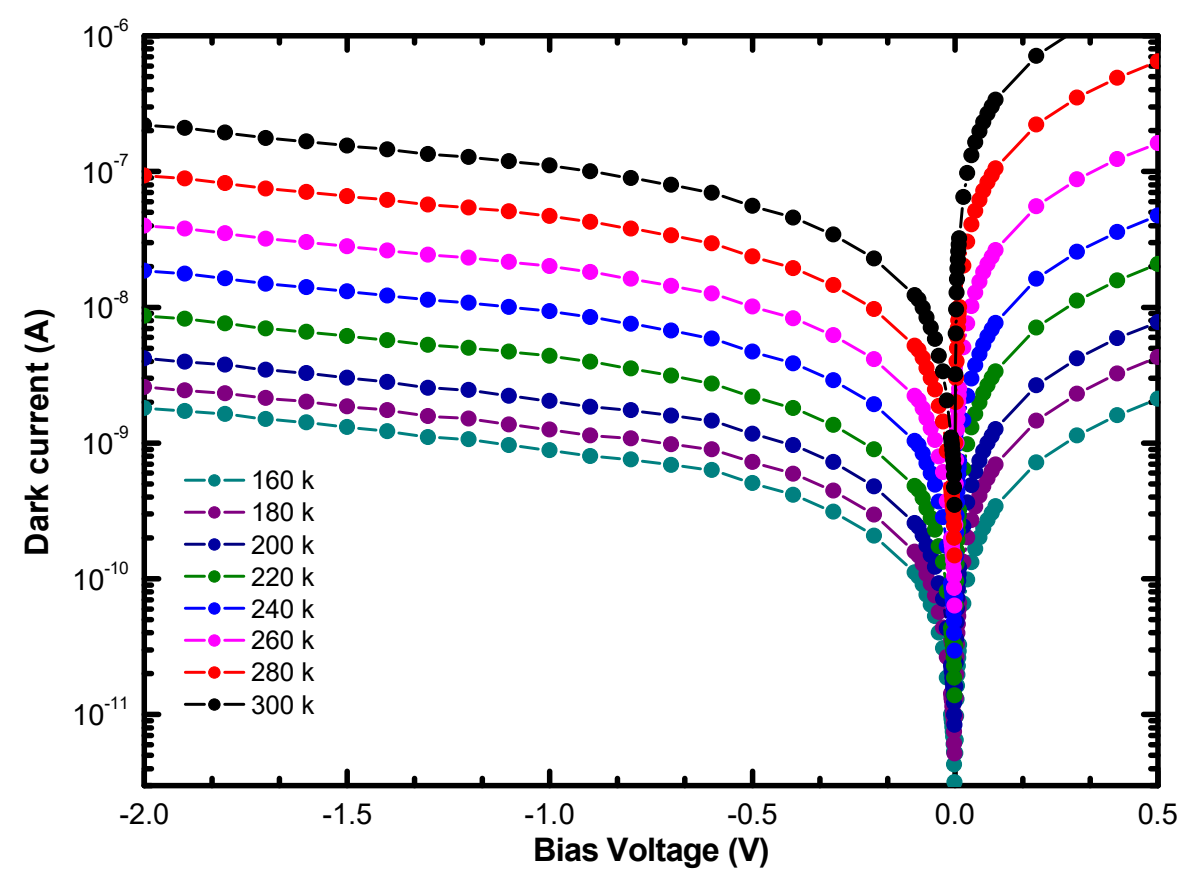

Figure 5 


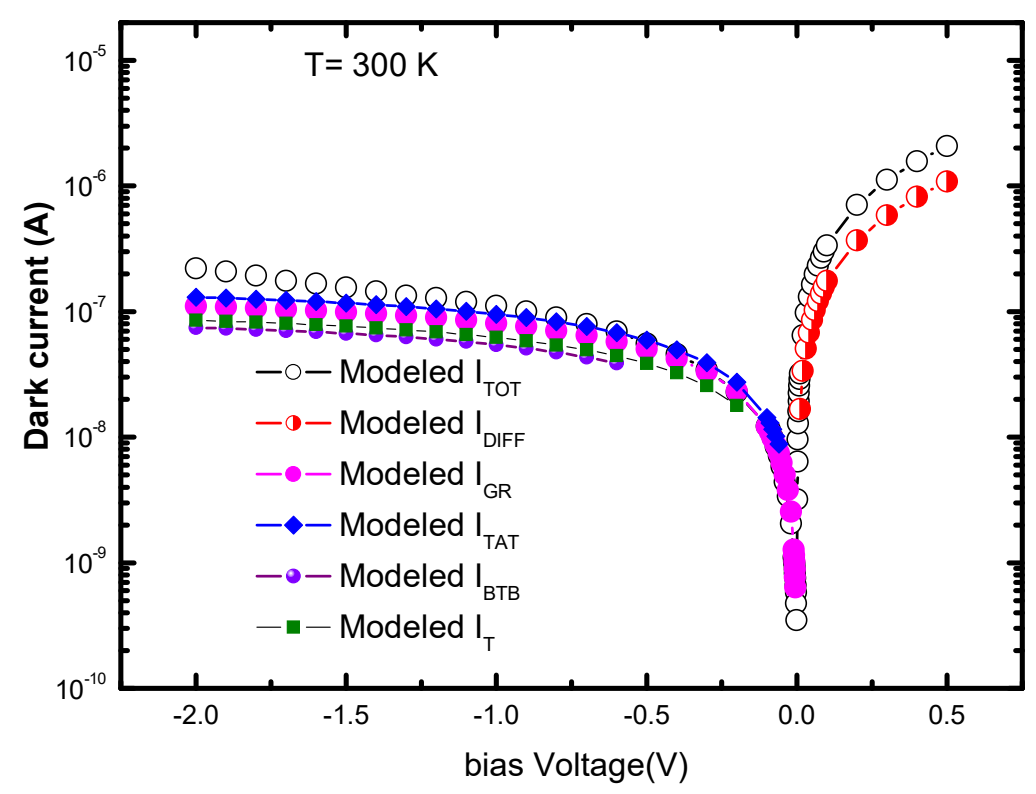

Figure 6

Temperature (K)

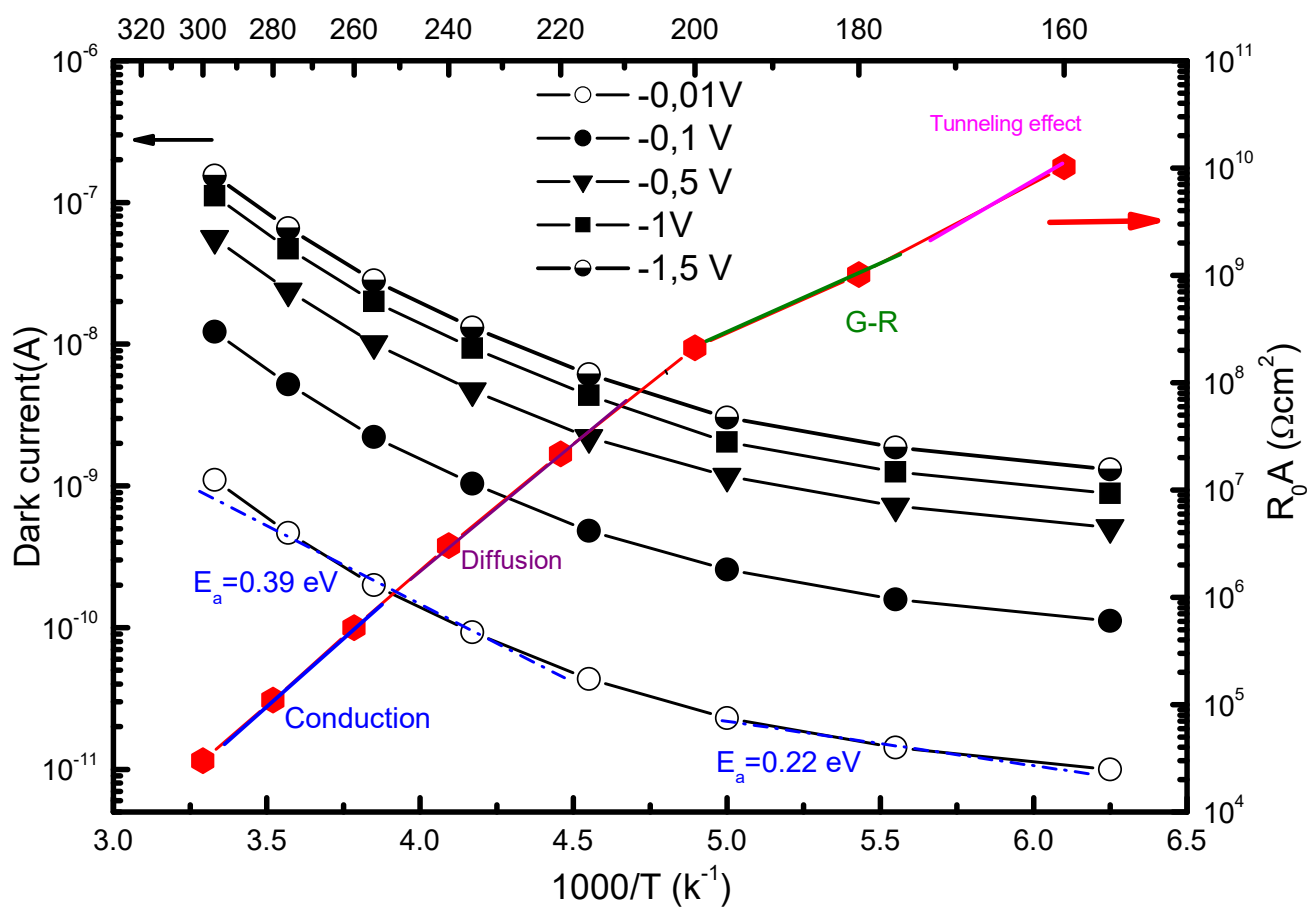

Figure 7 


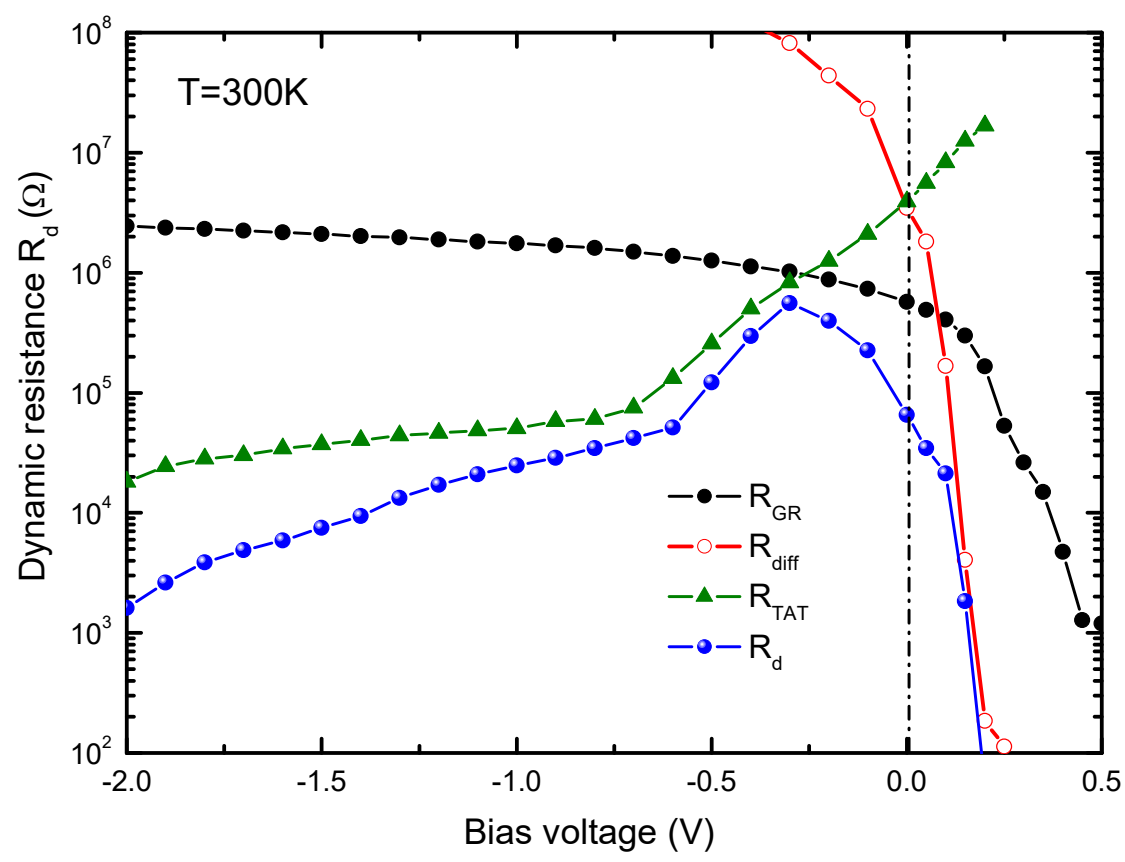

Figure 8

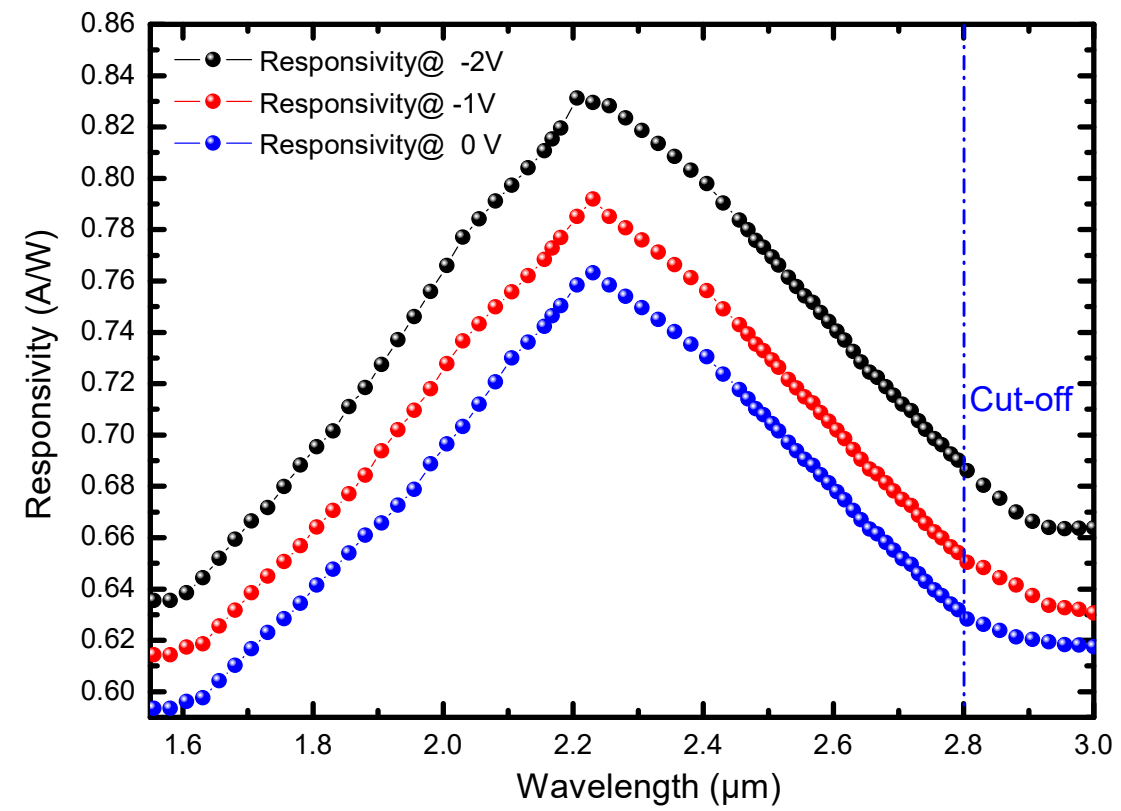

Figure 9 